Research Paper

\title{
Clinical Significance of Folate Receptor-positive Circulating Tumor Cells Detected by Ligand-targeted Polymerase Chain Reaction in Lung Cancer
}

\author{
Lin Wang ${ }^{1}$, Chuanyong $\mathrm{Wu}^{1}$, Lihua Qiao ${ }^{1}$, Wenjun $\mathrm{Yu}^{1}$, Qiaomei Guo ${ }^{1}$, Mingna Zhao ${ }^{1}$, Guohua Yang ${ }^{2}$, \\ Hang Zhao ${ }^{3 凶}$, Jiatao Lou ${ }^{1 凶}$ \\ 1. Department of Laboratory Medicine, Shanghai Chest Hospital, Shanghai JiaoTong University, Shanghai 200030, China. \\ 2. GenoSaber Biotech, 908 ziping Road, Bldg 26, Pudong, Shanghai, China. \\ 3. Department of Thoracic Surgery, Shanghai Chest Hospital, Shanghai JiaoTong University, Shanghai 200030, China. \\ $\triangle$ Corresponding authors: Hang Zhao, Shanghai Chest Hospital, Shanghai JiaoTong University, Shanghai 200030, China; Phone: 86-13701865603; Fax: \\ 86-21-22200000-2908; E-mail: h_zhao28@163.com; JiaTao Lou, Shanghai Chest Hospital, Shanghai JiaoTong University, Shanghai 200030, China; Phone: \\ 86-21-22200000-1601; Fax: 86-21-22200000-1601; E-mail: loujiatao@126.com. \\ (c) Ivyspring International Publisher. This is an open access article distributed under the terms of the Creative Commons Attribution (CC BY-NC) license \\ (https:// creativecommons.org/licenses/by-nc/4.0/). See http://ivyspring.com/terms for full terms and conditions.
}

Received: 2016.07.14; Accepted: 2016.09.30; Published: 2017.01.01

\begin{abstract}
Background: As the heterogeneity of CTCs is becoming increasingly better understood, it is clear that identifying particular subtypes of CTCs would be more relevant.

Methods: We detected folate receptor (FR)-positive circulating tumor cells (FR+ ${ }^{+}$-CTCs) by a novel ligand-targeted polymerase chain reaction (LT-PCR) detection technique.

Results: In the none-dynamic study, $\mathrm{FR}^{+}-\mathrm{CTC}$ levels of patients with lung cancer were significantly higher than controls (patients with benign lung diseases and healthy controls). With a threshold of 8.7 CTC units, FR ${ }^{+}$-CTC showed a sensitivity of $77.7 \%$ and specificity of $89.5 \%$ in the diagnosis of lung cancer. When compared with established clinical biomarkers including carcinoembryonic antigen (CEA), cytokeratin 19 fragment (CYFRA21-1), and neuron-specific enolase (NSE), $\mathrm{FR}^{+}$-CTC showed the highest diagnostic efficiency. Notably, the combination of FR ${ }^{+}-\mathrm{CTC}, \mathrm{CEA}$, NSE, and CYFRA21-1 could significantly improve the diagnostic efficacy in differentiating patients with lung cancer from benign lung disease. In our dynamic surveillance study, the CTC levels of 62 non-small cell lung cancer (NSCLC) patients decreased significantly after tumor resection.

Conclusion: We established a LT-PCR-based $\mathrm{FR}^{+}$-CTC detection platform for patients with lung cancer that exhibits high sensitivity and specificity. This platform would be clinical useful in lung cancer diagnosis and treatment response assessment.
\end{abstract}

Key words: circulating tumor cells; folate receptor; ligand-targeted polymerase chain reaction; lung cancer.

\section{Introduction}

Lung cancer is the primary cause of cancer-related death worldwide [1]. The majority of lung cancers are detected at an advanced stage in which treatments have limited efficacy and survival rates are dismally low. Therefore, detection and diagnosis of lung cancer at an early stage has the possibility of significantly reducing mortality with a greater chance of cure, which underscores the need for more effective, sensitive, and specific detection methods [2].

Circulating tumor cells (CTCs) were first observed from patients with metastatic malignancies more than 100 years ago [3]; however, it was not until the past few years that the clinical and research potential of CTCs became widely recognized. Studies have demonstrated that CTCs are important potential biomarkers for diagnosis, evaluation of treatment, and prognosis in several cancers including lung 
cancer [4]. More recently, accurate CTC detection has become increasingly important, especially when there is difficulty in obtaining sufficient tissue for diagnosis and molecular characterization in metastatic cancer.

While many challenges still exist, the detection of CTCs in blood is becoming increasingly feasible. To date, the majority of studies have employed the CellSearch $^{\mathrm{TM}}$ System (Veridex LLC, Huntingdon Valley, PA, USA) which is a semi-automated system for CTC quantification by employing magnetic beads coated with anti-epithelial cell adhesion molecule (EpCAM) antibodies. Other techniques involve EpCAM antibody-coated microporous chips, microporous polycarbonate filters, and PCR. However, methods based on EpCAM analysis have shown limitations in detecting CTCs in patients without or low epithelial characteristics [5-7]. Besides, CTC isolation by size may inadvertently omit cells less than $8 \mu \mathrm{m}$, thus requires further validation before clinical use [7]. Reverse transcriptase polymerase chain reaction (RT-PCR) can detect CTCs with high sensitivity [8]. However, post-transcriptional regulation that deregulates gene expression has been identified in numerous cancer cells [9], and it alters gene expression through modification of mRNA stability and/or transcriptional efficiency; thus protein content may possibly not correlate with mRNA levels.

Folate receptors (FRs) are cell-surface glycoproteins that are highly expressed in many cancers including, lung and ovarian cancer [10,11]. Our previous study showed promising clinical significance quantification of $\mathrm{FR}^{+}-\mathrm{CTC}$ s by a novel ligand-targeted polymerase chain reaction (LT-PCR) method [12]. Building on that previous study and in order to evaluate the efficacy and feasibility of $\mathrm{FR}^{+}$-CTC detection method, we conducted this large scale, prospective, single-center clinical trial.

\section{Materials and Methods}

\section{Patients}

From October 2013 to April 2014, we recruited 368 participants into this prospective, single-center clinical trial conducted at Shanghai Chest Hospital, Shanghai Jiaotong University, China. Among these patients, 197 patients with lung cancer, 119 patients with benign lung diseases and 52 healthy controls were enrolled in our none-dynamic study. In addition, 62 NSCLC patients among the 197 lung cancer patients who underwent surgical treatment were enrolled for our dynamic surveillance study. Lung cancer was defined on the basis of pathologic diagnosis, computed tomography (CT) scan, and laboratory examination according to the guidelines set forth by the National Comprehensive Cancer Network (NCCN). Benign lung diseases included pneumonia, tuberculosis, bronchiectasis or pneumothorax. The Research Ethics Committee of Shanghai Chest Hospital approved the clinical trial and an informed consent was obtained from each participant prior to study entry. The registration number of the clinical trial named "FR ${ }^{+}$CTC detection in lung cancer patients" is LS1308.

\section{CTC preparation and FA-oligonucleotide conjugation}

CTC analysis was performed using CytoploRare ${ }^{\circledR}$ circulating lung cancer cell kit provided by GenoSaber Biotech Co. Ltd. (Shanghai, China), as previously described [12]. We collected 3 $\mathrm{ml}$ of peripheral blood into EDTA-containing anticoagulant tubes from all study participants before treatment. Peripheral blood specimens were stored in $4-8^{\circ} \mathrm{C}$ and analyzed within $24 \mathrm{~h}$.

Following the manufacturer's instruction manual, CTCs were enriched by lysis of erythrocytes and immuno-magnetic depletion of leukocytes. The enriched CTCs were incubated with $10 \mu \mathrm{l}$ of labeling buffer that contained conjugates of a tumor-specific ligand, folic acid, and a synthesized oligonucleotide for 40 minutes at room temperature. Next, the unbound conjugates were washed off using $1 \mathrm{~mL}$ wash buffer and the cells were centrifuged at $500 \mathrm{rpm}$ for 10 minutes at $4^{\circ} \mathrm{C}$. The specific ligand-oligonucleotide conjugates were removed with $120 \mu \mathrm{L}$ stripping buffer for 2 minutes at $4^{\circ} \mathrm{C}$, collected by centrifugation, and neutralized by $24 \mu \mathrm{l}$ of neutralization buffer for further RT-PCR analysis.

\section{FR $^{+}$-CTC LT-PCR}

In the phase of PCR analysis, the specific conjugate was first annealed and extended on the RT primer before amplification. Then the extended conjugate was analyzed using a Taqman probe based on quantitative PCR method on ABI StepOne ${ }^{\mathrm{TM}}$ system (Life technologies). The primer sequences were listed as follows: reverse transcription (RT) primer (an oligonucleotide that is conjugated to the tumor-specific ligand folic acid), 5' CTCAACTGGTGTCGTGGAGTCGGCAATTCAGTT GAG- GGTTCTAA - 3'; forward primer, 5'-TATGATTATGAGGCATGA-3'; reverse primer, 5'-GGTGTCGTGGAGTCG-3'; TaqMan probe, 5'-FAM-CAGTTGAGGGTTC-MGB-3'. The following reaction conditions were used on the $\mathrm{ABI}$ StepOne ${ }^{\mathrm{TM}}$ instrument: denaturation at $95^{\circ} \mathrm{C}$ for 2 minutes, annealing at $40^{\circ} \mathrm{C}$ for 30 seconds, extension at $72^{\circ} \mathrm{C}$ for 30 seconds, and then cooling at $8^{\circ} \mathrm{C}$ for 5 minutes; 40 cycles of denaturation at $95^{\circ} \mathrm{C}$ for 10 seconds, 
annealing at $35^{\circ} \mathrm{C}$ for 30 seconds, and extension at $72^{\circ} \mathrm{C}$ for 10 seconds.

In this study, we used a self-defined CTC unit, which was the number of CTCs detected in $3 \mathrm{ml}$ blood, thus one CTC unit represented one CTC in $3 \mathrm{ml}$ blood. A serial of standards containing oligonucleotides $\left(10^{-14}\right.$ to $10^{-9} \mathrm{M}$, corresponding to 2 to $2 \times 10^{5} \mathrm{CTC}$ units $/ 3 \mathrm{~mL}$ blood) are used for CTC quantification. All patient samples were tested in duplicates with six standards and three quality controls.

\section{Clinical Tumor Biomarker Analysis}

Three milliliters of peripheral blood of all enrolled patients were withdrawn into coagulant tubes. After centrifuging at $800-1000 \mathrm{rpm}$ for $10 \mathrm{~min}$, the serum was collected for analysis of tumor markers (CEA, CYFRA21-1, NSE) by chemiluminescence method (Abbott Laboratories).

\section{Statistical Analysis}

Statistical analysis was performed using SPAA 18.0 software (SPSS Inc., Chicago, IL) or Prism 5.0 (GraphPad Software Inc., San Diego, CA). Wilcoxon test and Kruskal-Wallis test were used for comparison between groups, as appropriate. A receiver operating characteristics (ROC) curve was used to determine the threshold of specificity and sensitivity, and the area under the curves (AUC) was calculated for each index.

\section{Results}

\section{Patient characteristics}

We recruited 368 participants into this prospective, single-center clinical trial (Figure 1). Among these patients, 197 patients with lung cancer, 119 patients with benign lung diseases and 52 healthy controls were enrolled in our none-dynamic study (Table S1). In addition, 62 NSCLC patients among the 197 lung cancer patients who underwent surgical treatment were enrolled for our dynamic surveillance study. The clinical characteristics of the patients are presented in Table S2.

\section{Diagnostic value of $\mathrm{FR}^{+}-\mathrm{CTC}$ in patients with lung cancer}

To investigate the diagnostic value of $\mathrm{FR}^{+}-\mathrm{CTC}$, we compared CTC levels in patients with lung cancer, benign lung diseases and healthy donors. As shown in Figure 2A, the CTC levels in lung cancer patients (median 11.97 CTC units) was significantly higher than benign lung disease patients (median 6.72 CTC units, $p<0.0001$ ) and healthy donors (5.82 CTC units, $p<0.0001)$. However, there was no significant difference between the two control groups ( $p=$ 0.4355). The diagnostic value of $\mathrm{FR}^{+}-\mathrm{CTCs}$ in lung cancer patients was evaluated, and the area under curve (AUC) for FR-positive CTCs in discriminating between patients with lung cancer and the other two groups was 0.8607 (Figure 2B). According to the ROC analysis, the cut-off threshold between control group (benign patients and healthy volunteers) and the lung cancer group was 8.7 CTC units, with a sensitivity of $77.7 \%$ and specificity of $89.5 \%$. When compared with existing clinical biomarkers (CEA, CYFRA21-1, NSE), as shown in Table 1, the $\mathrm{FR}^{+}$-CTC detection method displayed the highest AUC and could satisfactorily discriminate lung cancer patients from benign lung disease and healthy controls. Notably, the combination of $\mathrm{FR}^{+}-\mathrm{CTC}$, CEA, NSE, and CYFRA21-1 significantly improved the diagnostic efficacy in differentiating patients with lung cancer from the other two groups (Figure 2C), implying that a multi-marker strategy integrating $\mathrm{FR}^{+}-\mathrm{CTC}$ levels with established tumor markers might be more effective for clinical lung cancer diagnosis.

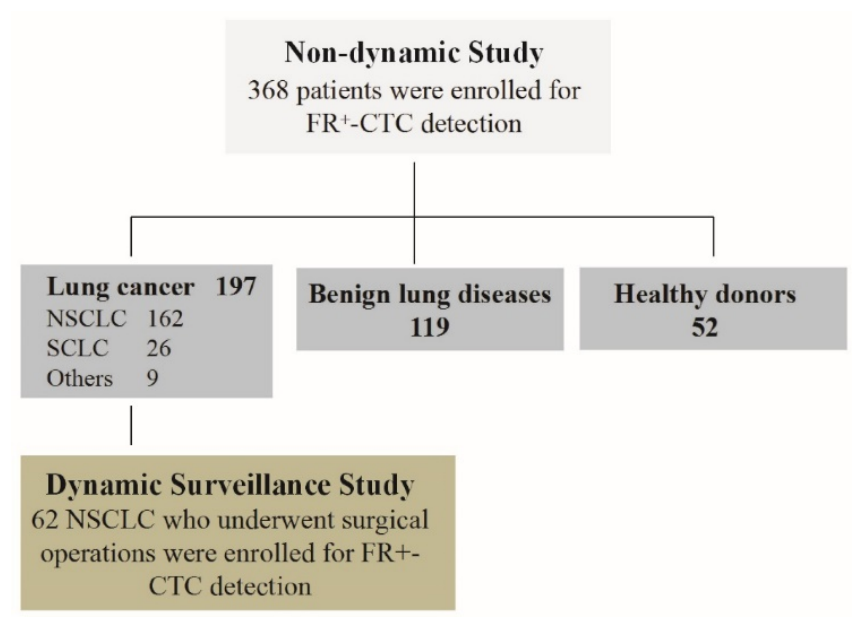

Figure 1. Distribution of patients enrolled in the study.

Table 1. Comparison of $\mathrm{FR}^{+}-\mathrm{CTC}$ with Established Tumor Biomarkers.

\begin{tabular}{ll}
\hline Lung cancer $v s$. benign disease & AUC $(95 \% \mathrm{CI})$ \\
\hline FR ${ }^{+}$-CTC & $0.7956(0.7205-0.8708)$ \\
CEA & $0.6289(0.5371-0.7208)$ \\
NSE & $0.5090(0.4120-0.6061)$ \\
CYFRA21- 1 & $0.5507(0.4551-0.6462)$ \\
CEA+NSE+CYFRA21-1 & $0.6495(0.5583-0.7406)$ \\
CEA+NSE+CYFRA21-1+ FR+-CTC & $0.8267(0.7601-0.8933)$ \\
\hline
\end{tabular}


A

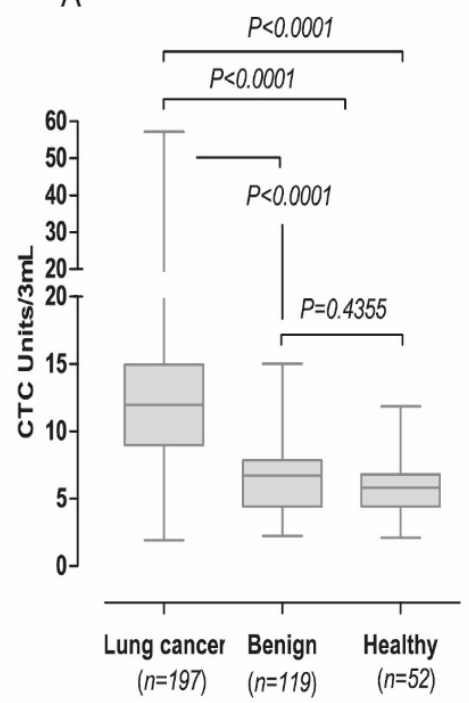

B

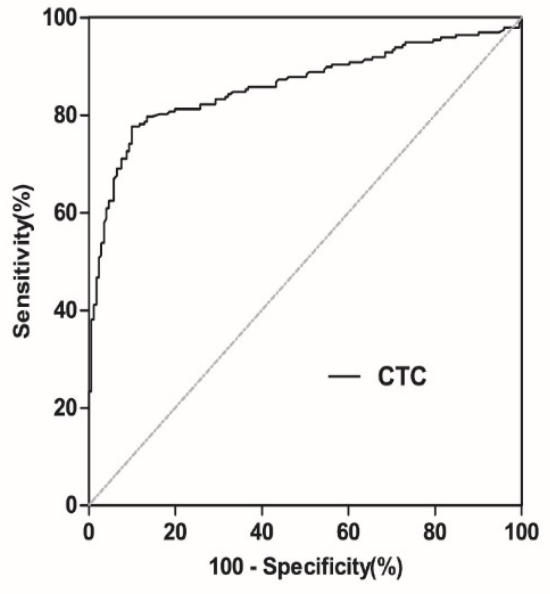

C

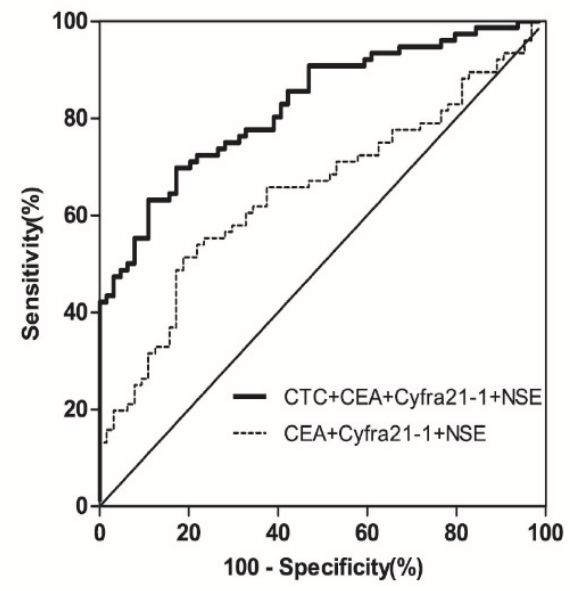

Figure 2. Diagnostic value of $F R^{+}-C T C$ s in patients with lung cancer. (A) $F R^{+}-C T C$ levels in patients with lung cancer, benign lung disease and healthy controls. (B) ROC curve for $F R^{+}-C T C$ in discriminating patients with lung cancer from patients with benign lung disease and healthy controls. (C) $R O C$ curve for FR+-CTC combined with tumor marker in discriminating patients with lung cancer from patients with benign lung disease and healthy controls.
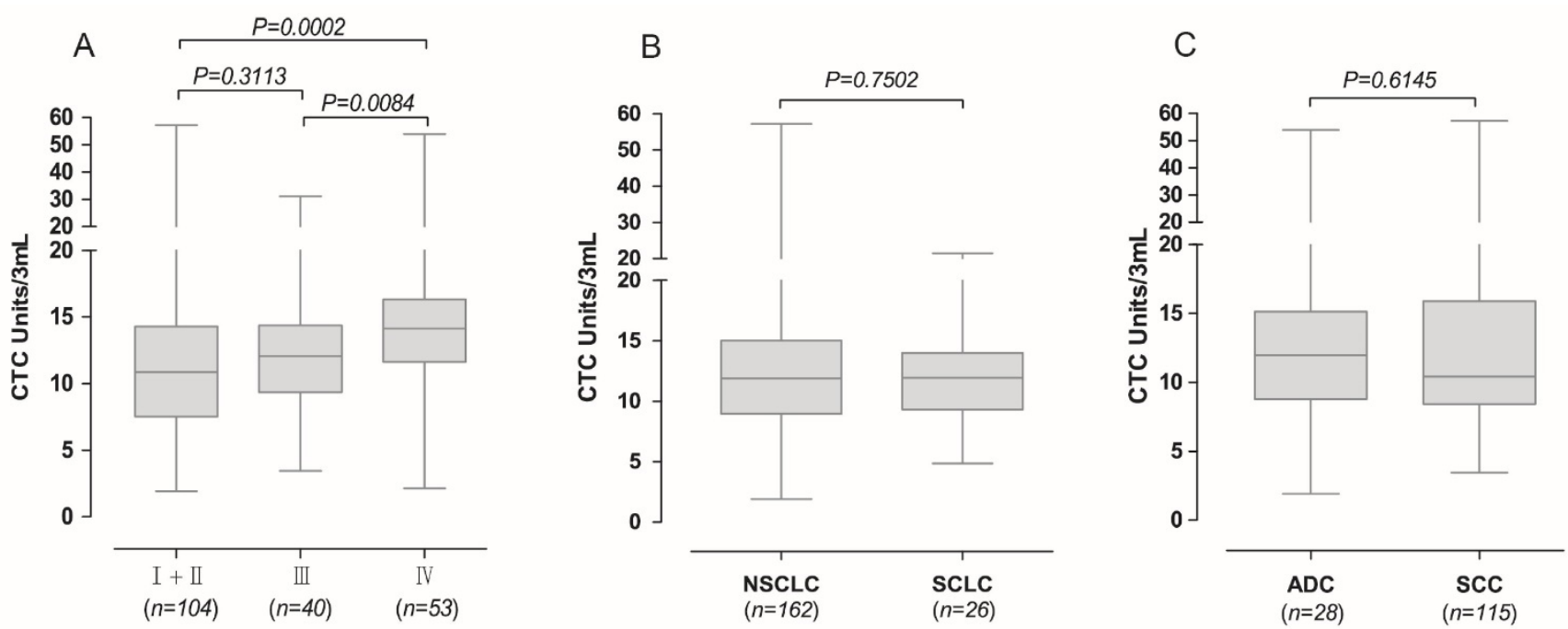

Figure 3. Association of $\mathrm{FR}^{+}-\mathrm{CTC}$ with TNM stage and histopathologic subtypes of lung cancer patients. (A) The FR+-CTC level in lung cancer patients with different TNM stages. (B) The FR+-CTC level between patients with NSCLC and SCLC. NSCLC, non-small cell lung cancer; SCLC, small cell lung cancer. (C) The FR+-CTC level between patients with ADC and SCC. ADC, adenocarcinoma; SCC, squamous cell carcinoma.

\section{Association of FR+-CTC with TNM stage and histopathologic subtypes of lung cancer patients}

We next examined whether $\mathrm{FR}^{+}$-CTCs could stratify TNM stages and histopathologic subtypes. Lung cancer stages were based on the seventh edition of the American Joint Committee on Cancer (AJCC) staging manual [13]. The CTC levels in patients with stage IV lung cancer were significantly higher than those with stage I and II lung cancer $(p=0.0002)$ and stage III lung cancer $(p=0.0084)$, while there was no significant difference among CTC levels in patients with stage I, II and III ( $p=0.3113$; Figure 3A). In assessing whether $\mathrm{FR}^{+}-\mathrm{CTC}$ could discern between
NSCLC and SCLC, the median levels in 162 NSCLC patients, (median 11.90) were not significantly different from the 26 SCLC patients (median 11.93) ( $p$ $=0.7502$, Figure 3B). Additionally, we did not find a significant difference in $\mathrm{FR}^{+}-\mathrm{CTC}$ levels in patients with adenocarcinoma (ADC) and squamous cell carcinoma (SCC) $(p=0.6145$, Figure 3C).

In a more detailed analysis, we investigated the diagnostic efficiency of $\mathrm{FR}^{+}-\mathrm{CTC}$ in different histological subtypes and pathological stages of lung cancer. As shown in Table 2, the diagnostic sensitivity for patients with stage I-IV lung cancer was $69.8 \%$ $(60 / 86), 72.2 \%(13 / 18), 80.0 \%(32 / 40)$, and $90.6 \%$ $(48 / 53)$, respectively. In regard to the different histological subtypes of lung cancer, the diagnostic 
sensitivity of the method for NSCLC and SCLC patients was $77.2 \%(125 / 162)$ and $80.8 \%(21 / 26)$, respectively.

These results suggested that patients with localized disease and those with metastatic lung cancer have distinct levels of CTC; however, the expression of folate receptor might be similar across all histological subtypes of lung cancer.

\section{Dynamic changes of $\mathrm{FR}^{+}-\mathrm{CTC}$ in patients with resectable NSCLC}

In a dynamic surveillance study, 62 NSCLC patients with CTC levels higher than 8.5 Units $/ 3 \mathrm{ml}$ before surgery were recruited to investigate the changes of $\mathrm{FR}^{+}-\mathrm{CTCs}$ during the peri-operative period. Blood samples were collected before and seven days after surgery (median $5.46 \quad \mathrm{FR}^{+}$-CTC Units/3ml for post-surgery; $12 \mathrm{FR}^{+}$-CTC Units/ $3 \mathrm{ml}$ for pre-surgery). As shown in Figure $4 \mathrm{~A}$ and $4 \mathrm{~B}$, compared with pre-surgery, $\mathrm{FR}^{+}-\mathrm{CTC}$ load decreased significantly after resection $(96.77 \%$ vs. $19.35 \%$, $p<0.0001)$. Among these patients, 48 patients with $\mathrm{FR}^{+}$-CTCs positive before resection achieved a negative status seven days after operation, 12 patients were still $\mathrm{FR}^{+}-\mathrm{CTC}$ positive, and two patients with $\mathrm{FR}^{+}-\mathrm{CTCs}$ negative before resection remained negative.

\section{Discussion}

Although CTCs were first observed more than 100 years ago, their clinical significance has only recently become widely recognized. Quantification and characterization of CTCs may serve as a surrogate diagnostic test that could constitute a "liquid biopsy" and provide real-time information about the patient's disease state $[14,15]$. Technology that can reliably identify CTCs in peripheral blood is becoming increasingly available. CellSearch ${ }^{\mathrm{TM}}$ System (Veridex LLC, Huntingdon Valley, PA, USA) has been by far the most commonly used method, and is the only technique thus far approved for routine use in the clinical setting by the FDA [16-18]. A recent method of CTC detection is "CTC-Chip" has been investigated for use in lung, prostate, pancreatic, breast, and colon cancer, and has been shown to reliably detect CTCs in $99 \%$ of patients with metastatic disease with a purity of approximately $50 \%$ [19]. Isolation of tumor cells by size is a non-immunological based, cytometric CTC isolation technique which has been used successfully to identify CTCs in a number of cancers such as breast, hepatocellular carcinoma, and NSCLC [20-22]. Though widely used, the antigen-dependent immunological techniques may sacrifice sensitivity for the sake of specificity and vice versa for non-immunological techniques. The heterogeneity of CTCs is becoming increasingly better understood and it is clear that identifying particular subtypes of CTCs would be more relevant.

Table 2. Diagnostic Efficiency of FR+-CTC in Lung Cancer Histopathologic Subtype.

\begin{tabular}{|c|c|c|c|c|}
\hline \multirow[t]{2}{*}{ Characteristics } & \multicolumn{2}{|c|}{$\begin{array}{l}\geq 8.70 \mathrm{FR}^{+}-\mathrm{CTC} \\
\text { Units } / 3 \mathrm{~mL}\end{array}$} & \multicolumn{2}{|c|}{$\begin{array}{l}<8.70 \mathrm{FR}^{+}-\mathrm{CTC} \text { Units } / 3 \\
\mathrm{~mL}\end{array}$} \\
\hline & NO. & $\%$ & NO. & $\%$ \\
\hline Lung cancer $(n=197)$ & 153 & 77.7 & 44 & 22.3 \\
\hline \multicolumn{5}{|l|}{ TNM stage } \\
\hline$I(n=86)$ & 60 & 69.8 & 26 & 30.2 \\
\hline II (n = 18) & 13 & 72.2 & 5 & 27.8 \\
\hline III $(n=40)$ & 32 & 80 & 8 & 20 \\
\hline $\mathrm{I}+\mathrm{II}+\mathrm{III}(\mathrm{n}=144)$ & 105 & 72.9 & 39 & 27.1 \\
\hline IV $(n=53)$ & 48 & 90.6 & 5 & 9.4 \\
\hline \multicolumn{5}{|l|}{ Histopathologic subtype } \\
\hline NSCLC (n = 162) & 125 & 77.2 & 37 & 22.8 \\
\hline $\operatorname{ADC}(n=115)$ & 88 & 76.5 & 27 & 23.5 \\
\hline $\operatorname{SCC}(n=28)$ & 20 & 71.4 & 8 & 28.6 \\
\hline Others of NSCLC $(n=19)$ & 17 & 89.5 & 2 & 10.5 \\
\hline $\operatorname{SCLC}(n=26)$ & 21 & 80.8 & 5 & 19.2 \\
\hline $\begin{array}{l}\text { Others of lung cancer }(n= \\
\text { 9) }\end{array}$ & 7 & 77.8 & 2 & 22.2 \\
\hline Benign diseases $(n=119)$ & 14 & 11.8 & 105 & 88.2 \\
\hline Healthy donors $(n=52)$ & 4 & 7.7 & 48 & 92.3 \\
\hline
\end{tabular}
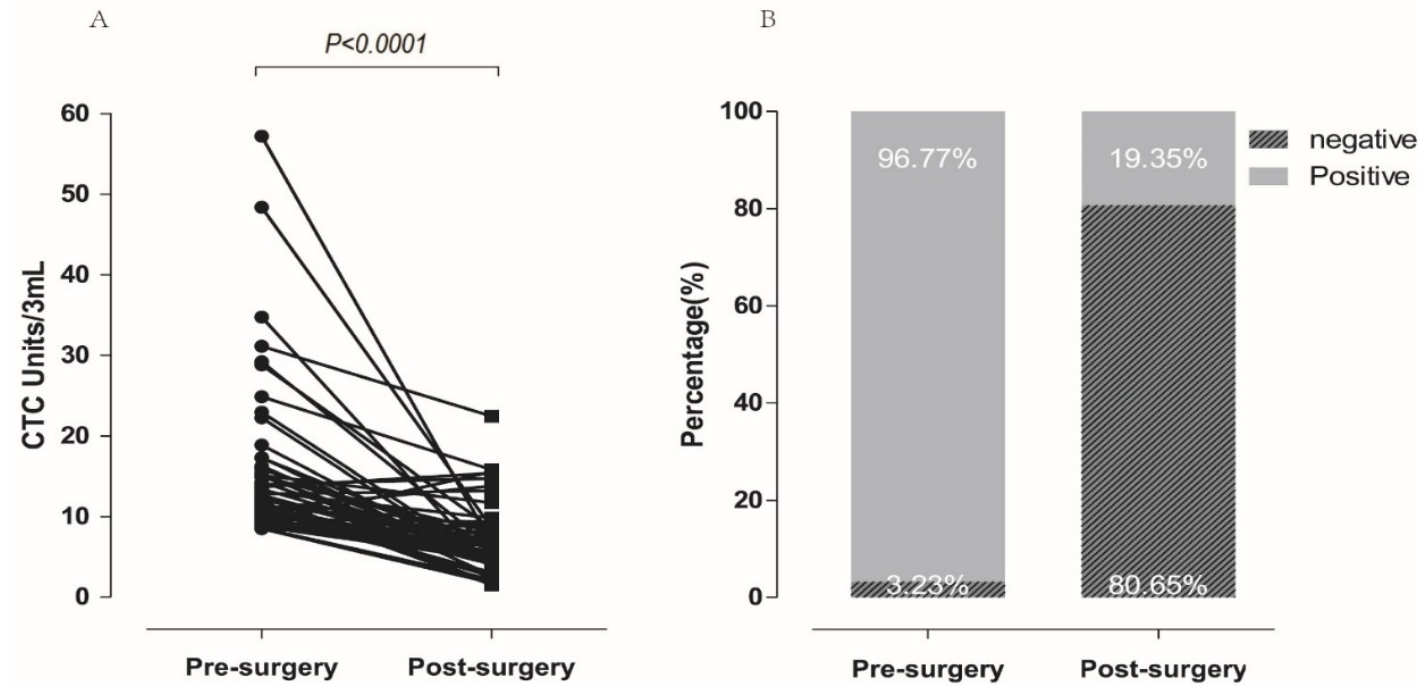

Figure 4. Dynamic changes of $\mathrm{FR}^{+}-\mathrm{CTC}$ in patients with resectable NSCLC. Dynamic changes of $\mathrm{FR}^{+}-\mathrm{CTC}$ level in patients receiving curative resection. $\mathrm{FR}^{+}$-CTC positive rates of patients before and after curative resection. 
The folate receptor (FR), a cell-surface receptor glycoprotein, which is highly expressed in a variety of cancers, especially in ovarian and lung cancers, has become an important potential drug target for patients with NSCLC $[10,23]$. Although FR has been identified in some normal tissues, including kidney, spleen and lung, no cells expressing FR have been identified in the circulatory system except for CTCs or activated monocytes $[10,24,25]$. Preliminary studies found that these activated monocytes subpopulation is barely detectable in blood samples from healthy donors or patients with benign disease [24,25]. Besides, FR expression is also found in tumor activated macrophages (TAMs) [27], and our previous study demonstrated that the depletion mechanism using anti-CD45 magnetic beads was sufficient to remove the impact of TAMs on CTC counts [12]. A study found that FR expression was upregulated in about $75.7 \%$ of patients with NSCLC [28], therefore, FR may be a potential target for detecting CTCs in patient with lung cancers.

Our previous small sample study showed promising clinical value of detecting $\mathrm{FR}^{+}-\mathrm{CTC}$ by a novel ligand-targeted polymerase chain reaction (LT-PCR) method in NSCLC patients [12]. LT-PCR is a sensitive, rapid, and cost-effective technique for molecular identification of CTC. The major drawback of this method is the relatively high false-positive rate due to contamination with leukocytes, which can be reduced by effective pre-enrichment of CTC. In order to validate our previous data and evaluate the feasibility of LT-PCR FR ${ }^{+}$-CTC detection method in the diagnosis of lung cancer, we conducted this large scale, prospective, clinical trial. We found that $\mathrm{FR}^{+}$-CTC levels were significantly higher in 197 patients with lung cancer compared with 119 patients with benign lung disease and 52 healthy donors $(p<0.0001$, Figure 2A). Using the marker of FR, our platform showed a significant diagnostic performance in discrimination between patients with lung cancer, patients with benign lung disease and healthy donors with an AUC of 0.8607 and a sensitivity of $77.7 \%$ and specificity of $89.5 \%$ (Figure 2B). In clinical practice, plasma tumor markers CEA, NSE, and CYFRA21-1 were used to help diagnose and surveil treatment efficiency of lung cancer. Comparing with these plasma tumor markers, $\mathrm{FR}^{+}-\mathrm{CTC}$ detection showed the highest $\mathrm{AUC}$ in the diagnosis of lung cancer (Table 1). Notably, the combination of $\mathrm{FR}^{+}$-CTC, CEA, NSE, and CYFRA21-1 could significantly improve the diagnostic efficacy in differentiating patients with lung cancer from benign lung disease (Figure 2C), implying that a multi-marker strategy integrating CTC levels with tumor markers might be a more effective method for clinical lung cancer diagnosis.
Moreover, our data also demonstrated the clinical significance of $\mathrm{FR}^{+}$-CTC detection by our LT-PCR platform for surveillance of treatment efficiency in patients with NSCLC treated with surgery (Figure 4A, 4B).

Studies on CTC detection in NSCLC previously identified a correlation between disease stages and CTC numbers [7], and our results also found that the CTC levels in patients with stage IV lung cancer were significantly higher than those with stage I and II lung cancer $(p=0.0002)$ and stage III lung cancer $(p=$ $0.0084)$, while there was no significant difference among CTC levels in patients with stage I, II and III ( $p$ $=0.3113$; Figure 3A). Although, previous studies demonstrated that the FR expression was much lower in SCC than in ADC in primary tumors [7,10,28,29], our results showed no significant difference of $\mathrm{FR}^{+}$-CTC levels between patients with SCC and NSCLC ( $p=0.6145$, Figure 3B), partly because gene expression between primary tumors and CTC samples is often different [30], as previously demonstrated in breast cancer [31].

In this large-scale, prospective clinical trial, we established a LT-PCR-based $\mathrm{FR}^{+}$-CTC detection platform for patients with lung cancer that exhibits high sensitivity and specificity. Our results indicate that $\mathrm{FR}^{+}-\mathrm{CTC}$ detected by this platform would be clinically useful in lung cancer diagnosis and treatment response assessment. Further investigation on the prognostic value of CTC levels detected by LT-PCR is required.

\section{Supplementary Material}

Supplementary tables.

http://www.jcancer.org/v08p0104s1.pdf

\section{Acknowledgement}

This work was supported by grants from the Ministry of Science and Technology of China (2014DFA33010), the Science and Technology Commission of Shanghai Municipality (14411950800, 13441902200), the Hospital Development Center of Shanghai (SHDC22014011), Shanghai Chest Hospital (2014YZDC10100, YZ14-22).

\section{Conflicts of Interest}

No potential conflicts of interest were disclosed.

\section{References}

1. Jemal A, Bray F, Center MM, et al. Global cancer statistics. CA Cancer J Clin. 2011; 61:69-90.

2. Oak $\mathrm{CH}$, Wilson $\mathrm{D}$, Lee $\mathrm{HJ}$, et al. Potential molecular approaches for the early diagnosis of lung cancer. Mol Med Rep. 2012; 6(5):931-936.

3. Ashworth TR. A case of cancer in which cells similar to those in the tumours were seen in the blood after death. Med J Australia. 1869; 14:146-149.

4. Plaks V, Koopman CD, Werb Z. Cancer, circulating tumor cells. Science. 2013;341(6151):1186-8 
5. Young R, Pailler E, Billiot F, et al. Circulating tumor cells in lung cancer. Acta Cytol. 2012; 56:655-660.

6. Gorges TM, Tinhofer I, Drosch M, et al. Circulating tumour cells escape from EpCAM-based detection due to epithelial-to-mesenchymal transition. BMC Cancer. 2012; 12:178.

7. Krebs MG, Sloane R, Priest L,et al. Evaluation and prognostic significance of circulating tumor cells in patients with non-small-cell lung cancer. J Clin Oncol. 2011; 29:1556-1563.

8. Ignatiadis $\mathrm{M}$, Xenidis $\mathrm{N}$, Perraki $\mathrm{M}$, et al. Different prognostic value of cytokeratin-19 mRNA positive circulating tumor cells according to estrogen receptor and HER2 status in early-stage breast cancer. J Clin Oncol. 2007; 25:5194-5202.

9. Audic Y, Hartley RS. Post-transcriptional regulation in cancer. Biol Cell. 2004; 96:479-498.

10. Parker N, Turk MJ, Westrick E, et al. Folate receptor expression in carcinomas and normal tissues determined by a quantitative radioligand binding assay. Anal Biochem. 2005; 338:284-293.

11. Christoph DC, Asuncion BR, Hassan B et al. Significance of folate receptor alpha and thymidylate synthase protein expression in patients with non-small-cell lung cancer treated with pemetrexed. J Thorac Oncol. 2013; 8:19-30.

12. Lou JT, Ben SQ, Yang GH, et al. Quantification of Rare Circulating Tumor Cells in Non-Small Cell Lung Cancer by Ligand-Targeted PCR. PloS One. 2013; 8(12): e80458.

13. Edge SB, Compton CC. The American Joint Committee on Cancer: the 7th edition of the AJCC cancer staging manual and the future of TNM. Ann Surg Oncol. 2010; 17: 1471-4.

14. Alix-Panabieres C, Pantel K. Circulating tumor cells: liquid biopsy of cancer. Clin Chem. 2013; 59:110-8.

15. Pantel K, Alix-Panabieres C. Real-time liquid biopsy in cancer patients: fact or fiction? Cancer Res. 2013; 73:6384-8.

16. Cristofanilli M, Budd GT, Ellis MJ, et al. Circulating tumor cells, disease progression, and survival in metastatic breast cancer. N Engl J Med. 2004; 351:781-91.

17. Cohen SJ, Punt CJ, Iannotti N, et al. (Pinzani et al., 2006). J Clin Oncol. 2008; 26:3213-21.

18. Bono JS, Scher HI, Montgomery RB, et al. Circulating tumor cells predict survival benefit from treatment in metastatic castration-resistant prostate cancer. Clin Cancer Res. 2008; 14:6302-9.

19. .Nagrath S, Sequist LV, Maheswaran S, et al. Isolation of rare circulating tumour cells in cancer patients by microchip technology. Nature. 2007; 450:1235-9.

20. Desitter I, Guerrouahen BS, Benali-Furet N, et al. A new device for rapid isolation by size and characterization of rare circulating tumor cells. Anticancer Res. 2011; 31:427-41.

21. Pinzani P, Salvadori B, Simi L, et al. Isolation by size of epithelial tumor cells in peripheral blood of patients with breast cancer: correlation with real-time reverse transcriptase-polymerase chain reaction results and feasibility of molecular analysis by laser microdissection. HumPathol. 2006; 37:711-8.

22. Vona G, Estepa L, Beroud C, et al. Impact of cytomorphological detection of circulating tumor cells in patients with liver cancer. Hepatology. 2004; 39:792-7.

23. Thomas A, Maltzman J, and Hassan R. Farletuzumab in lung cancer. Lung Cancer. 2013; 80:15-18.

24. He W, Kularatne SA, Kalli KR, et al. Quantitation of circulating tumor cells in blood samples from ovarian and prostate cancer patients using tumor-specific fluorescent ligands. Int J Cancer. 2008; 123: 1968-1973.

25. Reddy JA, Haneline LS, Srour EF, et al. Expression and functional characterization of the beta-isoform of the folate receptor on CD34(+) cells. Blood. 1999; 93: 3940-3948.

26. He $\mathrm{W}$, Wang $\mathrm{H}$, Hartmann $\mathrm{LC}$, et al. In vivo quantitation of rare circulating tumor cells by multiphoton intravital flow cytometry. PNAS. 2007; 104: 11760-11765.

27. Puig-Kroger A, Sierra-Filardi E, Dominguez-Soto A, et al. Folate receptor beta is expressed by tumor-associated macrophages and constitutes a marker for M2 anti-inflammatory/regulatory macrophages. Cancer Res. 2009; 69: 9395-9403.

28. Nunez MI, Behrens C, Woods DM, et al. High expression of folate receptor alpha in lung cancer correlates with adenocarcinoma histology and EGFR[corrected] mutation. J Thorac Oncol. 2012; 7:833-840.

29. O'Shannessy DI, Yu G, Smale R, et al. Folate receptor alpha expression in lung cancer: diagnostic and prognostic significance. Oncotarget. 2012; 3: 414-425

30. Rao CG, Chianese D, Doyle GV, et al. Expression of epithelial cell adhesion molecule in carcinoma cells present in blood and primary and metastatic tumors. Int J Oncol. 2005; 27:49-57.

31. Ligthart ST, Bidard FC, Decraene C, et al. Unbiased quantitative assessment of Her-2 expression of circulating tumor cells in patients with metastatic and non-metastatic breast cancer. Ann Oncol. 2013; 24: 1231-1238. 